PROGRESS REPORT

May, 1992

\title{
DIAGNOSTIC AND THERAPEUTIC APPLICATIONS OF DIODE LASERS AND SOLID STATE LASERS IN MEDICINE
}

\section{DE-FG05-91ER61226}

University of Texas M. D. Anderson Cancer Center

Steven L. Jacques, Ph.D. (PI)

DOE/ER/61.226--1

University of Texas at Austin

A. J. Welch, Ph.D.

University of Texas Medical Branch at Galveston

Massoud Motamedi, Ph.D.

Texas A\&M University

Sohi Rastegar, Ph.D.

Rice University

Frank Tittel, Ph.D.

Naval Research Laboratory

Leon Esterowitz, Ph.D.

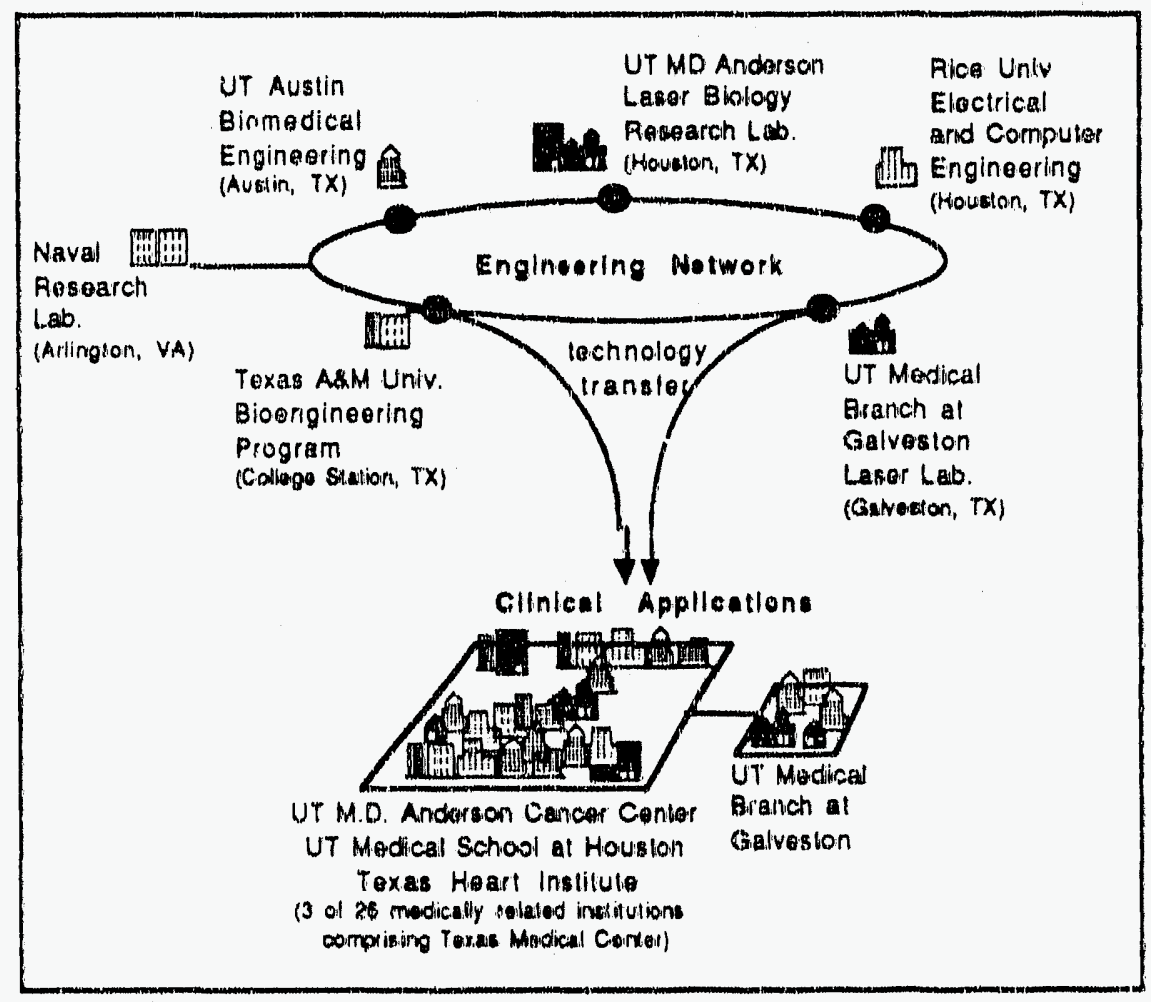




\section{Introduction}

The Texas Medical Center in Houston and the nearby UT Medical Branch at Galveston together constitute a major center of medical research activities. Laser applications in medicine are under development with the engineering assistance of the colloborating engineering centers at Rice University, UT-Austin, and Texas A\&M Univ. In addition, this collective is collaborating with the Naval Research Laboratory, where new developments in laser design are underway, in order to transfer promising new laser techrology rapidly into the medical environment.

Our research plan has not changed and we are proceeding along the schedule of the original plan. That research plan is summarized by the following description of DIAGNOSTICS and THERAPEUTICS, which appeared in the original proposal.

4.A.1 Refleciance spectroscopy

4.A.1.1 Bilirubinome'ry

4.A.1.2 Monitoring iv lines

4.A.1.3 Assessing body fluids

4.A.2 Fluorescence spectroscopy

4.A.2.1 Cerwical ca, dysplasias

4.A.2.2 Atherasclerotic plaque

4.A.2.3 Colonic ca

Brainca

Head/Neck ca

Lung ca

4.A.3 Raman spectroscopy

4.3.1 Cervical ca, dysplasias

4.3.2 Atherosclerotic plaque
UTMDA-LL,RICE,UTMISH-Ped

UTMDA-LL,RICE,UTMSH.PEd

UTMDA-LL,RICE,UTMSH-Ped

UTA, UTMDA-Gyn, UTMDA-ILL

UTA, UTMBG-Cardio

UTMDA-GIT, UTMDA-LL, UTA

UTMDA-Neuro, UTMDA-LL

UTMDA-HeadNeck, UTMDA-LL

UTMDA-Thoracic, UTMDA-LL

UTA, UTMDA-Gyn

UTA, UTMBG-Cardio, THI
4.B.1 Photocoagulation therapy

4.B.1.1 Prostate coagulation

4.B.1.2 Cyclophotocoagulation

4.B.2 Laser weiding

4.B.3 Cardiology

4.B.3.1 Angiosurgery

4.B.3.2 Photoacoustic relaxation

4.B.4 Pigmented lesion treatment by pulsed lasers

4.B.4.1 Prevention of melanoma

4.B.4.2 Clearance of deep lesions

4.B.5 Photodynamic Therapy (PDT)

4.B.5.1 solid-state laser PDT"

4.B.5.2 laser-diode array PDT
TAMU, UTMDA-LL, UTMDA-Urol, UTMBG-LL, UTMBG-Urol

UTA, NRL, UTMBG-LL, TAMU

UTA, NRL, UTMBG.LL, THI

TAMU, UTHI, UTMDA-LL, UTMBG-LL, THI, UTMDA-LL,

UTMDA-LL, UTMSH-DE'm, UTMBG-LL UTMBG-LL, UTMBG-Derm, UTMDA.LL

UTMDA-LL, UTMDA-VEAMEd, UTMBG-LL UTMDA-LL, UTMDA-VEtMed 
where the abbreviations in the above table are as follows:

$\begin{array}{ll}\text { UTMDA } & \text { University of Texas M. D. Anderson Cancer Center } \\ \text { UTA } & \text { University of Texas at Austin, Biomedical Engineering } \\ \text { UTMBG } & \text { University of Texas Medical Branch at Galveston } \\ \text { TAMU } & \text { Texas A\&M University, Bioengineering Program } \\ \text { RICE } & \text { Rice University, Electrical and Computer Engineering } \\ \text { NRL } & \text { Naval Research Laboratory } \\ \text { UTMSH } & \text { University of Texas Medical School at Houston } \\ \text { THI } & \text { Texas Heart Institute }\end{array}$

and the suffixes indicate various departments:

$\begin{array}{ll}\text { LL } & \text { Laser Laboratory } \\ \text { Ped } & \text { Pediatrics } \\ \text { Gyn } & \text { Gynecology } \\ \text { Cardio } & \text { Cardiology } \\ \text { GIT } & \text { Gastrointestinal Oncology and Digestive Diseases } \\ \text { Neuro } & \text { Neurosurgery } \\ \text { HeadNeck } & \text { Head and Neck Surgery } \\ \text { Thoracic } & \text { Thoracic Surgery } \\ \text { Urol } & \text { Urology } \\ \text { Derm } & \text { Dermatology } \\ \text { VetMed } & \text { Veterinary Medicine }\end{array}$

\section{Progress Reports}

In the following sections, progress reports are presented from each of the collaborating groups. Within each group's report, the interactions between groups are mentioned.

In several instances, reference is made to human studies. These studies are not officially a part of this DOE program, which does not cite human studies. In each case, these studies were conducted under approved human protocols. They represent our efforts to translate laser technology into the clinic. This DOE program supports the basic development and design efforts using animal and phantom tissue models which catalyze this technology transfer.

\section{UT M. D. Anderson Cancer Center}

Steven L. Jacques, Ph.D.

\section{A.1 Reflectance spectroscopy}

The goal of this project is to develop spectroscopic applications of diode lasers for the Neonatal Intensive Care Unit (NICU). We are currently conducting optical studies on prematurely newborn infants at the NICU at Herman Hospital, UTMSH-Ped (Dr. Oelberg). The method uses the optical fiber spectrophotometer and analysis algorithm described in Table 1 of the original proposal. More than 60 infants have been measured so far, and we now have a research nurse who is routinely measuring infants. We estimate the routine measurements will reach -50 infants per week over a 6-month period, or about 120 infants. The results of this work are to be applied in design of optical measurements that monitor three clinical situations:

1. quantifying hyperbilirubinemia (4.A.1.1)

2. monitoring iv lines (4.A.1.2)

3. assessingbody fluids (4.A.1.3) 
The aim is to establish design parameters and implement the optical techniques using diode laser sources.

We are also assisting RICE in development of another form of optical reflectance spectroscopy that utilizes diode lasers: Phase and Demodulation Spectroscopy. This topic is discussed in the RICE report.

\section{A.2.3 Fluorescence Spectroscopy}

The goal of this project is to develop endoscopic fluorescence spectroscopy as a means of locating and characterizing abnormal tissue sites, which is especially pertinent to screening for early cancer. We have begun our efforts by developing a prototype fluorescence bronchoscope in collaboration with UTMDA.Thoracic (Dr. Garret Walsh) borrowing an optical multichannel. analyzer (OMA) from UTMBG (Dr. M. Motamedi). The prototype system strives to meet two goals:

(1) Initial surveillance

The first step is to illuminate a large area with an excitation beam and use enhanced video images to locate abnormal fluorescence that indicates abnormal tissue sites.

(2) Local spectroscopic characterization

The second step is to obtain fluorescence spectra from specific sites using an optical fiber catheter. Spectroscopic signatures have potential for characterizing tissues sites more specifically than simple changes in the magnitude of fluorescence.

Our current prototype system uses our new OMA (not purchased by this program) and a helium-cadmium laser at $440 \mathrm{~nm}$ which achieves the high average power for the surveillance task but also can excite fluorescence spectra for local characterization. At this point, we have employed the HeCd laser with a 7-surrounding-1. fiber catheter to obtain spectra from local sites. This bronchoscopic system has measured the lungs of 19 human patients under an approved protocol which is not part of this DOE proposal. Therefore, this work should not actually be considered part of our DOE program. However, it does illustrate how our DOEsponsored program is catalyzing the transfer of technology to the clinic.

We have also implemented a nitrogen/dye laser system for tunable excitation of fluorescence spectra using a 7-surrounding-1 fiber catheter system. This system allows acquisition of the emission spectra for a series of excitation wavelengths. The resulting data array is called an excitation/emission matrix. We have measured such an excitation/emission matrix obtained in vivo from rat brain in collaboration with UTMDA-Neuro (Dr. Z. Gayan). We are measuring excitation-emission matrices from various cancer cell lines in cell culture and in tumors grown subcutaneously in rats. Such studies allow us to identify the optimum wavelengths for excitation and emission which can characterize a tissue site.

Our aim is to implement fluorescence spectra using pulsed solid state lasers. We are working with both the second and third harmonics (532 and $355 \mathrm{~nm}$ ) of the Q-switched Nd:YAG laser.

\section{B.1.1 Photocoagulation therapy: benign prostate hyperplasial (BPH)}

We are collaborating with UTMBG and TAMU on the development of diode laser coagulation of the prostate. Eventual collaboration with NRL will investigate the use of solid state lasers. We have obtained a diode laser bar from Lawrence Livermore National Laboratory (LLNL) and are developing a system for coupling the bar into a fiber bundle. Our 
goal is to establish a distributed light source about $1.5-\mathrm{cm}$ long which can be inserted into the urethra via an urethroscope. Current methods use the Nd:YAG laser from a single fiber, which leads to overheating of the site of irradiation and causes much vaporization. We estimate that as much as $87 \%$ of the applied energy is going into steam formation rather than into coagulation of the prostate. By distributing the laser energy source, the overheating can be mitigated.

We have also been chosen hy the Diomed Itd. of Cambridge, England, as a test site for their new 25.W diode laser system. This system is portable, self-contained, operates on 110 VAC wall outlet power, and delivers $25 \mathrm{~W}$ through a single $400 . \mu \mathrm{m}$ cole diameter fiber. We have purchased a fiber splitter (Canstar Inc., Canada) to split the $25 \mathrm{~W}$ into four $5 . \mathrm{W}$ fiber outputs. These four output fibers are being incorporated into a catheter system which uses a gold mirror to deflect the four fiber outputs at $90^{\circ}$ to yield a distributed irradiance.

We have modeled the optical propagation and thermal diffusion process in prostate for the 1064-nm NdYAG laser (and the $805-\mathrm{nm}$ diode laser) and concluded that current practitioners are attempting to achieve photocoagulation too rapidly. Rather than irradiate for $60 \mathrm{~s}$ at $60 \mathrm{~W}$, our modeling suggests that $180 \mathrm{~s}$ at $20 \mathrm{~W}$ (or $360 \mathrm{~s}$ at $10 \mathrm{~W}^{\prime}$ ) would achieve essentially the same spatial distribution of heat, and minimize the overheating of the surface. Therefore, we are developing and testing in dogs (initially at UTMBG and later at UTMDA) dosimetry protocols for optimal photocoagulation in the pros.ate.

Human studies of laser photocoagulation have begun at UTMDA.Urol (Dr. D. Johnson), under approved protocols which are not part of this DOE program. Again, the results of our animal testing under this DOE program are catalyzing the transfer of new laser applications into the clinic.

\section{B.3.1 Cardiology: angiosurgery}

In this project, we are testing the use of near-UV lasers, the third harmonic of the Nd:YAG laser $(355 \mathrm{~nm})$ and the doubled alexandrite laser $(365.400 \mathrm{~nm}$ ), to achieve tissue ablation. The eventual application is angiosurgery, however the application of laser ablation is quite broad. The choice of near.UV lasers is that protein absorption is utilized rather than water absorption as for the mid-IR lasers. Many have observed that multiple pulses from a mid. IR laser will dessicate tissue, lowering the tissue abosrption and interfering with the ablation process. In contrast, any dessication during multiple $355-\mathrm{nm}$ pulses will serve only to increase the absurption coefficient and enhance ablation. Therefore, the problem of dessication is selfcorrecting when near-UV wavelengths are used. Our preliminary firdings are that $355-\mathrm{nm}$ ablation is more efficient than anticipated on the basis of tissue optical properties at $355 \mathrm{~nm}$ under low-irradiance conditions. Therefore, we have been exploring the relative roles of (1) laser-induced increases of absorbance due to excited state absorption and (2) laser-induced spallation due to induced thermoelastic stress.

\section{B.3.2 Cardiology: photoacoustic relaxation}

The goal of this project is to use laser-induced acoustic pressure waves to cause relaxation of blood vessels. Our progress so far has been implementation of a sputtered lithiumniobate detector for measurement of laser-induced pressure waves. The very thin detector allows very high frequency response. We have been studying the acoutic signals generated in bovine liver, chicken breast, and dog prostate by the 355,532,630, and $1064 \mathrm{~nm}$ wavelengths of a Q-switched Nd;YAG/harmonic crystal/dye laser system. The thermoelastic expansion generated by the laser propagates through the tissue sample to the rear surface where the 
detector is located. We have experimentally established the effects of geornetry on such photomechanical measurements, for example how do beam size, tissue thickness, and optical penetration interact. We have used the technique to measure the absorption and reduced scattering coefficients of the tissues, which constitutes a new optical method similar to classical photoacoustic spectroscopy but applicable to scattering medium. We have also implemented the theory of laser-induced stress wave propagation for comparison with the experimental results. In summary, we have established the behavior of laser-induced acoustic waves in tissues, both theoretically and experimentally. We are poised to intiate experiments on blood vessel relaxation using our dorsal rat skin window chamber model which allows real-time video recording of the vessels during the laser irradiation.

\section{B.4 Pigmented lesion treatment by pulsed lasers}

This project has not yet been initiated. However, we have established a collaboration with Dr. M. Levy at Texas Childrens Hospital where pulsed dye lasers are used for treatment portwine stain lesions. Dr. Levy and Dr. Jacques (UTMDA) are preparing a human protocol for investigation of $\mathrm{Q}$-switched second-harmonic $\mathrm{Nd}$ :YAG laser treatment of superficial pigmented lesions. The UTMDA-LL intends to conduct experimental tests in animals and phantom tissues to elucidate the proper dosimetry for such treatments. The human studies are not part of this DOE program, but illustrate how our DOE program is actively catalyzing the transfer of laser technology into the clinic.

\section{B.5 Photodynamic Therapy (PDT)}

We have been conducting PDT on several cancer cell lines grown as subcutaneous tumors in the flanks of rats using the Nd:YAG/harmonic crystal/dye laser $(630 \mathrm{~nm})$ and two porphyrin sensitizers: (1) Photofrin II from Quadralogic Technologies Inc, Canada, and (2) protoporphyrin IX (PPIX) whose de novo synthesis in cells in stimulated by administration of the metabolic precusor $\delta$-aminolevulenic acid (5-ALA). We are quantifying the degree of dye saturation and dye photodestruction caused by the high-irradiance Q-switched pulsed laser exposure in comparison to low-irradiance continuous light exposure. Our preliminary results show a 3-fold lower efficiency of PDT using the pulsed laser system, suggesting saturation effects. Future work will be investigating the optimal duty cycle (on-off light exposure) which allows reperfusion of oxygen into the cancer cells from nearby blood vessels during the off cycle.

We are using fluorescence spectroscopy to monitor the rate constants for PPIX de novo synthesis in cell cuitures and animal tumors, and the rate constant for photodestruction during laser irradiation. Our preliminary results are guiding development of dosimetry protocols that optimize PDT using PPIX. We are considering the logistics of implementing such PPIX.PDT for treatment of prostate cancer and breast cancer.

We are collaborating with UTMGB-LL to obtain and test new diode lasers from an industriai source that will generate light in the $630-\mathrm{nm}$ region suitable for PDT.

\section{UT at Austin}

A. J. Welch, Ph.D.

\section{A.2.1 Diagnosis of Cervical Pre-cancer or Dysplasia}

The goal of this project is to develop a spectroscopic technique to better direct biopsy placement during colposcopic screening for cervical dysplasia. We have conducted studies to determine which excitation wavelengths are most promising for this work. We have recorded fluorescence excitation emission matrices of 16 samples of normal and neoplastic human 
cervical tissue in vitro. Following spectroscopic interrogation, samples were submitted for histologic analysis. A preliminary analysis of the data indicates that $360 \mathrm{~nm}$ excitation is a promising wavelength for discriminating normal, inflammatory and neoplastic tissue. We conducted fluorescence microscopy studies to identify the morphologic structures responsible for this fluorescence. At this excitation wavelength, collagen fibers in the stroma and cosinophils in the epithelium and stroma contributed significant fluorescence in normal, inflammatory and neoplastic tissues. In inflammatory and neoplastic tissues, eosinophilic infiltration was more marked. In normal and inflammatory tissues, no significant fluorescence was observed from epithelial cells. However, carcinoma in situ was associated with a significant epithelial cell fluorescence.

To aid in deconvolving tissue spectra at $360 \mathrm{~nm}$ excitation, we determined the fluorescence properties of eosinophils. Eosinophils were isolated from human whole blood. Fluorescence excitation emission matrices were measured, and an excitation emission maxima was observed at $360 \mathrm{~nm}$ excitation, $420 \mathrm{~nm}$ emission. the fluorescence of serum dwelling eosinophils appeared visually different than that of tissue dwelling eosinophils. At $360 \mathrm{~nm}$ excitation, tissue dwelling cells exhibited a yellow fluorescence, while serum dwelling cells exhibited bluish fluorescence. We are currently investigating the reasons for this difference.

We are presently building a prototype system to record fluorescence spectra from human cervix in vivo. We are constructing a biopsy forceps with an attached optical fiber probe to measure fluorescence emission spectra from normal and abnormal appearing areas at colposcopy. Initial studies will be conducted at $360 \mathrm{~nm}$ excitation based on the results of the preliminary studies described above.

\section{A.2.2 Examination of the Atherosclerotic Process at the Molecular Level}

Our ultimate goal in this project is to develop a combined spectroscopic and ultrasonic imaging system to determine the chemical composition and morphological structure of atherosclerotic plaque in vivo. We will utilize this system to image coronary artery atherosclerosis in symptomatic patients to predict in advance the least invasive, most effective form of therpay. Two major advances were made in the past year.

1) At UTA we have constructed a prototype imaging system which is capable of rccording spectroscopic and ultrasonic images of coronary artery in vitro. This system consists of a mechanical ultrasound catheter with a torroidally shaped piezo-electric transducer. The transducer sends and receives $20 \mathrm{MHz}$ ultrasound pulses to the arterial wall via a rotating concave mirror at a $45^{\circ}$ angle with respect to the lumenal axis. The piezo-electric transducer can either be coupled to a pulser-receiver to provide A mode ultrasound scans from pixels of interest or to a commercially available ultrasound imaging system (CVIS, Sunnyvale, CA) available at UTMBG to provide B mode images of the arterial cross section. An optical fiber bundle is epoxied into the central hole of this transducer and uses the same mirror to deliver excitation light to the tissue and conduct resulting fluorescence to a spectrograph coupled to an optical multi-channel analyzer. We are presently collecting the first combined spectroscopic and ultrasonic tissue images with this system. In the next year, we will use the system to measure spectroscopic and ultra-sonic images of atherosclerotic tissue removed from patients undergoing atherectomy at UTMBG. Following imaging, histochemical analysis of vessels will be performed. Patients will be followed and algorithms will be developed to relate the frequency of restenosis to features of the spectroscopic and ultrasonic images. IRB approval has recently been approved for this project at UTMBG and is pending at UTA. Currently, the 
spectroscopic portion of this system operates at $476 \mathrm{~nm}$ excitation. Spectral data are analyzed using algorithms previously developed to quantify the amount of structural protein, ceroid and oxy-hemoglobin.

2) In an attempt to expand the scope of chromophores which can be probed spectroscopically with this system, we have conducted a study of the fluorescence properties of the structural proteins collagen and elastin both in situ in human aorta and extracted from human placenta. We have recorded fluorescence excitation emission matrices from these proteins and compared our results to those reported in the literature. Our conclusions are that type I collagen, type III collagen and elastin have distinct spectroscopic properties in the UV and visible regions of the spectrum. Fluorescence is observed from the aromatic amino acid tyrosine and from aromatic crosslinks (pyridinoline in collagen and desomosine in elastin). In addition, collagen III exhibits a unique fluorescence peak at $500 \mathrm{~nm}$ excitation not found in type I collagen; the molecular origin of this fluorescence is unknown at this time. Based on the results of this study, we will develop algorithms to identify the proportions of type I and type III collagen and elastin from fluorescence spectra of arterial tissue at 320 and $500 \mathrm{~nm}$ excitation. This will be incorporated in the imaging system described above.

\section{B.1.2 Cyclophotocoagulation}

In vitro optical properties of the conjunctiva, sclera, and combined ciliary muscle and pigmented ciliary epithelium were measured for the pig eye as a function of wavelength. Published data for the absorption coefficient for monkey and rabbit pigment epithelium were employed to deconvolve the absorption and scattering coefficients for just the ciliary muscle. A Monte Carlo simulation of light transport provided estimates of the rate of heat generation in each layer at 800 and $1060 \mathrm{~nm}$.

A preliminary experiment was conducted in the in vivo rabbit eye to compare treatments at $860 \mathrm{~nm}$ and $1060 \mathrm{~nm}$. Power and exposure time were varied to demonstrate the effect of these parameters. Resulting lesions were examined histologically at UTMBG.

In vivo lesions produced during cyclophotocoagulation cannot be directly observed. To circumvent this problem, the iris has been removed from a rabbit to provide direct visualization of the coagulation process during diode laser irradiation. The laser irradiation portion of this experiment will be conducted on May 12, 1992.

During the next 12 months, additional data will be obtained to better define optical properties and in vivo experiments will be conducted to measure temperature profiies at the conjunctiva and at the ciliary epithelium. Irradiation parameters and contact delivery will be investigated for laser diode cyclophotocoagulation.

\section{B.2. Laser Welding}

Constant temperature vessel welding was conducted using in vitro saphenous veins. Five $\mathrm{mm}$ longitudinal incisions were welded with radiation from a shutter-controlled argon laser. A $\mathrm{PbSe}$ detector measured tissue temperature and a signal closed the laser shutter whenever a preselected temperature was exceeded. The bursting strength of the welded saphenous veins was determined for 30 sarnples. The average bursting pressure of welds at temperatures from $100^{\circ}$ to $120^{\circ} \mathrm{C}$ were $80 \%$ higher than welds at temperatures from $70^{\circ}$ to $90^{\circ} \mathrm{C}$. 
During the year a $10 \mathrm{~W} \mathrm{CW}$ Ho and Thuliurn:YAG laser was installed to investigate tissue welding at 2.01 and $2.1 \mu \mathrm{m}$. On May 10 a Thulium rod from NRL will be added to our argon laser-Ti:Sapphire system to simulate diode pumping of the Thulium rod. These new lasers will be integrated with a temperature feedback system to weld in vitro saphenous veins and in vivo canine vessels. An IR fiber will transmit 3.11 $\mu \mathrm{m}$ radiation to a $\mathrm{HgCdTe}$ detector for real time temperature feedback.

Next year NRI will add a grating to our CW Ho-Thulium laser to provide turning over the 2 $\mu \mathrm{m}$ water absorption peak.

\section{B.5 Photodynamic Therapy}

We are developing a technique based on steady state fluorescence spectroscopy to measure the concentration of photosensitizing drugs in vivo. To initiate this project, we have characterized the steady state fluorescence and absorption properties of Photofrin II over the excitation wavelength range $300-550 \mathrm{~nm}$ and the emission wavelength range $310-700 \mathrm{~nm}$ for a variety of concentrations and solvent conditions. These in vitro studies have shown that both the molar extinction coefficient and fluorescence quantum yield are very dependent on the concentration of the Photofrin, decreasing with increasing concentration. It is hypothesized that this is due to an aggregation of the Photofrin at higher concentrations. This work suggests that quantifying Photofrin II concentration from absorption or steady state fluorescence measurements will be difficult. However, other studies have suggested that unaggregated species in tissue are responsible for the primary PDT effect. As the quantum yield of unaggregated species is higher than that of aggregated species, fluorescence may prove valuable in predicting the extent of the PDT effect. To test this hypothesis we are utilizing a window chamber model containing implanted tumors. Using this chamber in rats, fluorescence excitation emission matrices have been recorded by attaching a fiber optic catheter to the face of the window. We have demonstrated that the fluorescence intensity can be monitored as a function of time with this method. Future studies will involve treating tumors with PDT and correlating the cell killing to the observed fluorescence e intensity prior to treatment. In addition, similar studies will be conducted with other photosensitizers.

\section{UT Medical Branch at Galveston}

Massoud Motamedi, Ph.D.

\section{B.1. PHOTOCOAGULATION}

\section{Prostate photocoagulation: Benign Prostate Hyperplasia}

Benign Prostatic Hyperplasia (BPH) is a significant health problem for primarily elderly men, many of whom have major pre existing medical problems. Based on autopsy studies, about $60 \%$ of men have evidence of prostatic hyperplasia at age $61-70$, and about $50 \%$ of men of the same age will present with clinical signs and symptoms of BPH. Annually approx ately 500,000 surgical procedure are performed to treat $\mathrm{BPH}$. In order to reduce the morbidity oi the surgical treatment and to offer acceptable therapy to high risk patients, the potential role of non-surgical treatment of BPH has been recently considered. Recent applications of laser for this treatment suggest that this procedure causes less patient traurna, feiver complications, a shorter hospital stay, and can be performed much faster than current techniques.

Over the past several year, we have investigated the influence of various parameters in the thermal response of in vitro dog prostate to Nd:YAG laser irradiation. Temperature thresholds for tissue coagulation and ablation, and depth of therma! injury were determined. 
Ten dog prostates were irradiated at 18 spots. An infrared camera was used to monitor tissue temperature at the irradiated surface or at a plane cut axially to the beam to observe depth of diffusion. Temperature measurements were correlated to histologic changes. Laser irradiation was delivered at 20 watts ( 5 seconds to 120 seconds) with spot sizes of $2 \mathrm{~mm}$ and $10 \mathrm{~mm}$.

Tissue coagulation began at $60^{\circ} \mathrm{C}$. Specimens heated to $150^{\circ} \mathrm{C}$ demonstrated a high degree of dehydration and superficial coagulation. Ablation onset was consistently announced by an explosion at $150{ }^{\circ} \mathrm{C}$. Carbonization occurred at $200^{\circ} \mathrm{C}$. 'This and higher temperatures $\left(>200^{\circ} \mathrm{C}\right)$ were reached quickly during irradiation in air, leading to carbonization and pyrolysis of the exposed urethral layer. If laser irradiation was stopped at this point, heat diffusion and thermal damage was confined to the urethra and the superficial prostate. The confining effect of the urethral mucosa was overcome by maintaining a layer of saline over the urethral surface during irradiation or by removing the urethra prior to laser exposure, thus allowing for much deeper heat penetration and thermal injury.

This in vitro study has provided valuable information in the investigation of the thermal response of canine prostate to $\mathrm{Nd}$ :YAG laser irradiation. The importance of surface cooling to allow deeper heat penetration and thermal injury to occur was appreciated. These in vitro observations will aid and enhance the efficacy and the success of our in vivo canine studies.

Results of in vivo studies of ten mongel canines with palpable prostates revealed that high doses were effective in achieving tissue penetration of $5.9 \mathrm{~mm}$. In most cases tissue explosion took place followed by some degree of carbonization and narrow crater formation. The popcorn effect was often accompanied by bleeding. The performance of the laser probe was greatly compromised during the irradiation period, gradually delivering much lower laser powers. On the other hand, at the low dose, equal to superior lesion depth were achieved (0.9-1.1) without experiencing any explosions and bleeding. The integrity of the laser probe was also conserved.

\section{Application of High Power Diode Laser for Photocoagulation of Myocardium}

Both supraventricular and ventricular tachyrrhythmias are important clinical problems often requiring pharmacologic or non-pharmacologic therapy for intolerable symptoms, hemodynamic instability, or the potential for sudden cardiac death. Ventricular tachycardia (VT) and fibrillation (VF) are primarily responsible for 300,000 to 400,000 cases of sudden cardiac death in the United States each year. Thi= project is designed to assess the feasibility, safety, dosimetry, and potential long-term sequellae of diode la ier ablation of normal and chronically infarcted ventricular myocardium, and to test for the first time a "smart" catheter for diode laser delivery.

To assess light distribution within myocardium, optical properties were determined using integrating sphere and a spectrophotometer to ineasure total reflection and transmission of thin sections $(100-1200 \mu \mathrm{m})$ of fresh canine myocardium in collaboration with UTMDA-LL. An inverse solution to transport theory was used to estimate the absorption and effective scattering coefficients at 810 and $1064 \mathrm{~nm}$ (diode and Nd:YAG laser respectively). These coefficients and reported coefficients for blood are listed in table 1. This study supports our initial hypothesis that the differences in optical properties of tissue at 810 and $1064 \mathrm{~nm}$ are not significant and one could adjust the irradiation parameters to obtain the same rate of myocardial heating using either laser. 
Table 1: Optical properties of dog heart, and whole human blood (40\% Hct)

\begin{tabular}{|l|l|l|l|l|}
\hline \multicolumn{1}{|c|}{ Tissue } & \multicolumn{1}{|c|}{$\begin{array}{c}\text { Wavelength } \\
(\mu \mathrm{m})\end{array}$} & \multicolumn{1}{c|}{$\begin{array}{c}\text { Absorption } \\
(1 / \mathrm{cm})\end{array}$} & $\begin{array}{c}\text { Scattering } \\
(1 / \mathrm{cm})\end{array}$ & \multicolumn{1}{c|}{$\begin{array}{c}\text { Penetration } \\
(\mathrm{mm})\end{array}$} \\
\hline Heart & 810 & 1.0 & 4.1 & 2.6 \\
\hline & 1064 & 0.85 & 2.8 & 3.3 \\
\hline Oxy-blood & 810 & 4.5 & 6.6 & 2.2 \\
\hline & 1064 & 3.0 & 3.4 & 3.3 \\
\hline
\end{tabular}

To investigate the utility of high-power laser diode for coagulation of cardiovascular tissue and to establish the equivalency criteria of 810 laser diode and $1064 \mathrm{~nm} \mathrm{Nd}$ :YAG lasers, the DioMed diode laser $(810 \mathrm{~nm})$ was used to irradiate fresh beef myocardium The tissue response was studied based on (1) thermographic analysis of surface temperature, (2) gross evaluation of lesions, and (3) histological evaluation of tissue response.

An Inframetric infrared camera was used to monitor the surface temperature during irradiation. To study the influence of laser power and rate of tissue heating on the tcmperature rises in myocardial tissue, three different power settings were usted. In this study the laser radiation was delivered via a $600 \mu \mathrm{m}$ fiber in a non-contact mode with a spot size of $6 \mathrm{~mm}$. The results support our hypothesis that temperature rises as high as $90^{\circ} \mathrm{C}$ can be easily achieved using the high power diode laser delivered via fiber optic catheter.

Since Nd:YAG laser has been recognized as the laser of choice, comparison was made between the thermal response of fresh beef myocardial tissue to diode laser vs. Nd:YAG laser radiation. Using a similar fiber optic catheter and delivering the same incident energy simultaneously while monitoring the surface temperature rise in both spots using an infrared camera, we observed that the rate of temperature rise for the diode laser is slightly higher than that associated with application of Nd:YAG laser irradiation. Table 2 shows the comparison between lesion size made under saline or in air and measured grossly. The histology of the lesions induced by diode laser is similar to those reported for Nd:YAG laser.

Table 2. Comparison of myocardial tissue response to diode and Nd:YAG laser

\begin{tabular}{|l|l|l|l|l|l|}
\hline $\begin{array}{l}\text { Laser } \\
\text { Source }\end{array}$ & $\begin{array}{l}\text { Power } \\
(\mathrm{W})\end{array}$ & $\begin{array}{l}\text { Time } \\
(\mathrm{Sec})\end{array}$ & $\begin{array}{l}\text { Lesion } \\
\text { Diameter }\end{array}$ & Lesion Depth & Medium \\
\hline Diode & 5 & 30 & $3.3 \mathrm{~mm}$ & $5.3 \mathrm{~mm}$ & saline \\
\hline Nd:YAG & 5 & 30 & 1.0 & $3.3 \mathrm{~mm}$ & saline \\
\hline Diode & 3 & 60 & $2.3 \mathrm{~mm}$ & $5.3 \mathrm{~mm}$ & saline \\
\hline Nd:YAG & 3 & 60 & $<1 \mathrm{~mm}$ & $1.3 \mathrm{~mm}$ & saline \\
\hline Diode & 5 & 10 & $3.8 \mathrm{~mm}$ & $4.0 \mathrm{~mm}$ & air \\
\hline Nd:YAG & 5 & 10 & $2.0 \mathrm{~mm}$ & $1.5 \mathrm{~mm}$ & air \\
\hline Diode & 3 & 10 & $3.3 \mathrm{~mm}$ & $2.8 \mathrm{~mm}$ & air \\
\hline Nd:YAG & 3 & 10 & not visible & not visible & air \\
\hline
\end{tabular}

In summary, our preliminary results suggest that it is possible to achieve a superior or at least comparable tissue response using high power diode laser.

\section{Development of Feedback for Real Time Monitoring of Tissue Coagulation}

Increasingly lasers are used to photocoagulate tissue. To improve the safety and efficacy of laser therapy, it is critical to develop a means for assessing in real time the extent of tissue 
involvement and laser-induced thermal damage. Our aim has been to test the feasibility for using high frequency ultrasound imaging to monitor lesion growth during laser irradiation of tissue and eventually develop a smart laser system. It is expected that the high spatial resolution associated with application of high frequency ultrasound imaging would enable us to use ultrasonograph for precise mapping of tissue damage and thus optimize the results of laser therapy.

We have developed a combined laser-ultrasound catheter to monitor the changes in tissue echogencity during high power laser irradiation. Ultrasound catheters with 20 or $30 \mathrm{Mhz}$ transducers from CVIS (Cardiovascular Imaging Systems) were combined with a modified optical fiber tip to monitor the tissue response and deliver the laser beam, respectively. The ultrasound catheter has a rotating mirror that scans a plane transverse to the catheter axis over $360^{\circ}$. The fiber optic catheter allowed for delivery of the laser beam at a $90^{\circ}$ angle with respect to the axis of the optical fiber. Both ultrasound catheter and optical fiber were put together side by side so that both ultrasound and laser beams were applied on the same plane. Fresh beef myocardium and canine prostate were irradiated using Nd:YAG laser beam delivered via a 400 $\mu \mathrm{m}$ fiber. Laser powers of 30 to $40 \mathrm{~W}$ were delivered for various exposure times.

Increased tissue echogenicity was observed during laser irradiation, especially while imaging with the $30 \mathrm{MHz}$ catheters. Reversible and irreversible changes were observed. The reversible changes seemed to be related to transient temperature rises in the tissue. After laser irradiation, permanent changes indicating deeper tissue echogenicity tended to correlate well with depth of tissue coagulation. In the case of the images obtained by using the $30 \mathrm{MHz}$ transducers, which provided the best resolution, increased echos were observed for lesions as deep as $8 \mathrm{~mm}$.

The results of this study suggest that high frequency ultrasound imaging could be useful for monitoring depth of tissue damage during laser irradiation.

\section{B.4. TREATMENT OF PIGMENTED LESIONS BY PULSED LASERS}

In the past six months we have been studying the applications of pulsed solid state lasers for treatment of pigmented skin lesions. Studies conducted in the past few years have shown that for the first time short pulsed Q-switched ruby lasers can be used to effectively remove black tattoo with no scarring. These findings have generated significant interests in medical community with emphasis on understanding the mechanism for the tissue response as well as optimizing the treatment parameters to allow for removal of tattoo and other pigmented lesions. In this study we are determining the optimum laser radiation and exposure time for treatment of various tattoos by examining the effects of wavelength and pulsed duration on the response of normal tissue and tattoos containing various pigments. Furthermore, we are performing standard spectrophotometric studies to determine the absorption characteristics of dyes that are used for tattooing and use various Q-switched lasers including Alexandrite, Nd:YAG, and ruby lasers to treat normal and tattoo targets in animal models. The animal model used in this study include ginnie pigs and mini-pigs. Studies are being analyzed based on, 1) acute and chronic response of tissue, and 2) morphologic and histologic analysis of tissue sections using light and electron microscopy to cietermine the extent of laser-induced thermal and mechanical injury in tissue. 


\section{A.2. FLUORESCENCE SPECTROSCOPY}

Collaborative arrangement has been established between UTMB, UTMD, UTA and Dr. Dick Sterenborg from the Laser Center in Amsterdam. The Netherlands, to develop a spectroscopic technique for early detection of melanoma based on techniques such as in vivo NAIH fluormcence measurements. Preliminary data on excitation-emission maps of normal subjects have been collected. The detection system consists of a CCD camera, OMA, and nitrogen laser will be assembled in the summer of 1992 when Dr. Sterenborg will start his year of sabbatical research working with investigators from UTMB, UTMD, and UTA.

\section{Education and Training:}

a. Training of Bhysician and Residence

Dr. Tom Cammack, who is becoming the chief residence at the Division of Urology at UTMB next year, has spent the last nine months working on the project reiated to development of smart 'ascr catheter for treatment of benign prostate hyperplasia (BPH, 4.B.1.1). Dr. Cammack has been trained in use of laser for prostate coagulation and application of Ultrasound Imaging fur monitoring tissue response real time by performing in vitro as well as in vivo studies. He has recently completed a training course for clinical use of lasers and will be participating in clinical trials of laser therapy for BPH. This study will start in July of 1992.

Dr. Susan Dozier who is fourth year residence in the department of Dermatology at UTMB has been working on the use of pulsed laser for removal of tattoo and other pigmentations in skin (4.B.4). She has been trained to use Q-switched Alexandrite and Nd:YAG lasers in an animal model.

b. Training of Graduate Students

As part of our training effort, Bahman Anvari, a Ph.D. candidate at the Bioengineering Program of TAMU has been working in the Laser Laboratory at UTMB on developing a computer model to predict tissue response during photocoagulation of p:nstate. The model will enable us guide the animal studies and account for variables such as prestate geometr $y$ and the effects of blood perfusion on the laser induced thermal field during laser therapy. This work should further allow us to investigate the feasibility for development of a smart laser catheter for treatment of benign prostate hyperplasia. Some of the preliminary results of this research have already been presented and additional work has been submitted.

\section{Tecbnology Transfer}

In the past year we have developed a very active working relation with number of laser and catheter companies that are interested in biomedical applications of photonics technology. These companies have benefited from our efforts in studying the potential use of ingh power diocle lasers for tissue coagulation (i.e. Diomed, Inc., Abela Laser System, Johnson \& Johnson, McDonnell Douglas) as well as use of high power solid state laser for prostate coagulation (i.e. Trimedyne Inc.)

\section{List of Abstracts and Proceedings:}

a. Proceedings:

1. Photocoagulation of Myocardial Tissue: A Comparative Study on the Effects of High Power Diode $(810 \mathrm{~nm})$ and $\mathrm{Nd}$ Y YAG Laser.

Massoud Motamedi'1, Steven L Jacques2 ${ }^{2}$, Sohi Rastegar ${ }^{3}$, SPIE Vol, 1642, 1992

1. The University of Texas Medical Branch, Galveston, Texas

2. The University of Texas MD Anderson Cancer Center, Houston, 
Texas

3. Texas A\&M University, College Station, Texas

2. Liver Coagulation with $810 \mathrm{~nm}$ Diode Laser and NdiYAG Laser.

Steven L Jacques' ${ }^{1}$, Sohi Rastegar2, Massoud Motamedi3', SPIE Vol. 1646, 1992

1. The University of Texas MD Anderson Cancer Center, Houston, Texas

2. Texas A\&M University, College Station, Texas

3. The University of Texus Medical Branch, Galveston, Texas

3. Equivalency of $810 \mathrm{~nm}$ Diode Laser \& $1064 \mathrm{~nm} \mathrm{Nd:YAG} \mathrm{Laser} \mathrm{Interaction} \mathrm{with} \mathrm{Tissue.}$

Sohi Rastegar", Stever: L Jacques², Massoud Motamedi3, SPIE Vol. 1646, 1992

1. Texas A\&M University, College Station, Texas

2. The University of Texas MD Anderson Cancer Center, Houston, Texas

3. The University of Texas Medical Branch, Galveston, Texas

\section{b. Abstracts}

1. Endoscopic laser treatment of prostatic hyperplasia: optimizing irradiation parameters for maximum coagulation depth. J.T. Cammack, M. Motamedi, J.H. Torres, E. Orhuela, D. Cowan, M.M. Warren. South Central Section of American Urological Association, 1992

2. Response of canine prostate to Nd:YAG laser irradiation: Thermographic analysis and histologic correlation James T. Cammack, Massouc' Motamedi, Jorge H. Torres, Eduardo Orihuela, Michael M. Warren. Department of Surgery and the Sealy and Smith Biomedical Laser Laboratory of the Biomedical Engineering Center University of Texas Medical Branch at Galveston. Tenth Annual Meeting on Biornedical Engineering Research in Houston, 1992.

3. Application of high frequency ultrasound imaging for monitoring tissue damage during laser irradiation. Jorge H. Torres*, Massoud Motamedi, James T. Cammack, Alfred Johnston, and Daniel Hendrix. Sealy ind Smith Biomedical Laser \& Ultrasonics Laboratories of the Biomedical Engineering Center and tic Department of Surgery, University of Texas Medical Branch at Galveston. Tenth Annual Meeting on Biomedical Engineering Research in Houston, 1992.

4. Modeling of intraluminal heating of issuc by laser and by contact heating: Implications for prostate treatment. B. Anvari, M. Motamedi, S. Rastepar. Bioengineeririg Program, Texas A \& $M$ University, University of Tex\% Medical Branch at Galveston. Tenth Annual Meeting on Biomedical Engineering Research in Houston, 1992.

5. A New Modality for the Treatment of Benign Prostatic Hyperplasia. Bahman Anvari ${ }^{1}$, Massoud Motamedi ${ }^{2}$, Sohi Rastegar ${ }^{1}$, and James T. Cammack, 1 Bioengineering Program, Texas A\&M University, College Station, TX. ${ }^{2}$ Division of Urology, University of Texas Medical Branch, Galveston, TX. (submitted to IEEE.EMBS, 1992) 
The activities for the first phase of this research involved experimental as well as theoretical work. Two Ph.D. student's, three M.S. student's, and one undergraduate's research projects at Texas A\&M have been/are related to this work. Two graduate students and one undergraduate were supported during the first phase of this project. One of the Ph.D. students is spending a year at UTMB in Galveston under this grant. One master's thesis has been completed, a copy of abstract and acknowledgment of which is enclosed.

\section{COLLABORATIONS}

Collaborative research were done, as planned, with UT-MDA, UTMB, UT-Austin and THI (Texas Heart Institute) for this project. This involved several trips by the PI and his students to Houston and Austin to perform experimental studies and discuss theoretical developments. Communication between the investigators as well as supervision of remotely located students is being done through electronic mail, computer netwrork two-way ('talk') communication, telephone, FAX, and regular visits.

\section{STUDIES}

\section{B.1 Photocoagulation}

A diode laser $(810 \mathrm{~nm})$ was loaned to the group by DIOMED, Inc., and used for experiments on several tissues in Houston and in Austin. Thermographic studies using a thermal camera were done in Austin for simultaneous comparison of diode and Nd:YAG lasers.

Theoretical studies involved the use of a SUN 3/60 computer at Texas A\&M and two SPARC-II Workstation at UTMB ( directly and by remote access). Studies were done in computer simulation of light and temperature distribution by Monte Carlo, finite element, finite difference, and analytical methods. A dynamic damage model was developed to estimate the extent of damage under varying optical properties. These programs were used for analysis of damage in lasers irradiate tissue under various irradiation conditions. In particular the programs were used for comparison of Nd:YAG and diode laser coagulation of prostate, liver, and myocardium, based on optical properties measured at UTMDA-LL. The details of methods and finding are found in the publications resulting from these studies. Some of the main finding are hichlighted below.

\section{Experimental studies:}

- Many similarities were observed in coagulation of tissue between diode and Nd:YAG lasers. Diode lasers have distinctly differing spatial profiles and laser spot behavior as a function of distance from the fiber is different than that observed with the Nd:YAG laser. It is important to be aware of this behavior when treating tissues with diode lasers.

- Studies of coagulation in egg-white with the addition of prescribed absorber and scatterer concentration proved to be a useful technique for simulating and analyzing coagulation under varying optical properties, a phenomenon which for a given biological tissue occurs as a function of "ravelength.

- Two-layer studies were done which showed that under this geometry the coagulation front moves away from the interface into the thermally weaker layer, regardless of direction of irradiation. 
- Starting with a given absorber concentration, a reduction in light transmission was simulated by either addition scatterer or further addition of absorbers. This resulted in totally different results in both optically and thermally. Measurement of optical attenuation alone is an insufficient predictor of the reaction of tissue to laser irradiation. Full optical properties must be measured for proper dosimetry.

\section{Theoretical studies:}

- A finite element code was linked to a monte-carlo program for simultaneous calculation of light and temperature distributions in tissue.

- A theory for dynamic analysis of damage was developed in collaboration with UTMDA. IL and applied in the above programs.

- The dynamic damage inodel was used in studying coagulation of myocardium, liver, and prostate. The temperature time history may have an increasing slope followed by decreasing slope depending on the scattering albedo of the native and denatured tissue under consideration. In this regard liver and myocardium behaved differently.

- In some cases the simulation of surface temperature rise for diode and Nd:YAG lasers predicted different behavior compared to the observed experimental results. The mismatch may be attributed to the measurements being done on different tissues than those on which optical properties were measured. It is important for these analyses to simultaneously measure the optical properties and irradiate for coagulation or ablation. This policy was exercised in simulation studies in egg-white coagulation and results were consistent.

- Presence of blood may in particular have a varying effect in Nd:YAG versus diods laser coagulation of tissue.

\section{B.3.1 Cardiology: angiosurgery}

A Ho:YAG/Er:YAG lasers was loaned to Texas A\&M by the THI, on which several basic studies were performed on ablation of tissue.

\section{Experimental studies:}

- Water is the known dominant chromophore for ablation of tissue in the Mid-IR range. It was found that the hydration level may play an important role in ablation of vessel walls for the Ho:YAG laser but not for the Er:YAG laser. In comparison with UV-lasers, it was found that the Ho:YAG laser is more effective in removing calcified plaques from the arteries.

\section{Theoretical studies:}

- Comparison of ablation rates between Excimer and Ho:YAG lasers was studied and results were in agreement with experimental observations.

- The role of mass diffusion and dehydration in the course of laser irradiation of tissue was theoretically developed in collaboration with UTMDA.IL and is being implemented.

- A user friendly program for analytical simulation of laser ablation in an absorbing tissue was developed for the Macintosh computer. 


\section{PUBLICATIONS/PRESENTATIONS}

The following conference proceedings and presentations were given about the research activities of this grant:

- S. Rastegar, S. L. Jacques, M. Motamedi, and B. Kim, "Analysis of Equivalency of High-Power Diode Laser $(810 \mathrm{~nm})$ and Nd:YAG Laser $(1064 \mathrm{~nm})$ for Coagulation of Tissue," SPIE Proceedings, Vol. 1646, 1992.

- S. Rastegar, B. Kim, and S. L. Jacques, "Role of Temperature Dependence of Optical Properties in Laser Irradiation of Biological Tissue," SPIE Proceedings, Vol. 1646, 1992.

- S. S. Kumbhani, Simulation of Optical Absorption and Scattering in Gel and Egg White," Master's Thesis, Texas A\&M University, May 1992.

- K. Kadipasaoglu, S. Rastegar, F. J. Clubb, Jr., L. Moreau, J. L. Conger, C. R. Reichart, W. D. Hare, R. Matsuwaka, N. Furusho, O. H. Frazier, "Acute and Chronic Resi onse of Normal Canine Endothelium to Ho:YAG Laser Irradiation," Presented at 12th Annual Meeting of American Society for Lasers in Medicine and Surgery, Lake Bueno Vista, FL, May 17-19, 1992; also submitted for publication in Lasers is Surgery and Medicine, 1992.

- B. Anvari, M. Motamedi, and S. Rastegar, "Modeling of intraluminal heating of tissue By laser and by contact heating: Implications for prostate treatment," Tenth Annual Biomedical Engineering Research Conference in Houston, March 19-20, 1992

- B. Edelen, S. Rastegar, M. Moore, and K. Kadipasaoglu, "Studies of UV and Mid-IR Laser Ablation of Tissue," Tenth Annual Biomedical Engineering Research Conference in Houston, March 19-20, 1992

- B. Kim, S. Rastegar, S.L. Jacques, "Role of Temperature Dependence of Optical Properties in Laser Irradiated Tissue," Tenth Annual Biomedical Engineering Research Conference in Houston, March 19.20, 1992

-S. S. Kumbhani and S. Rastegar, "Simulation of Absorption and Scattering and in Gel and Egg White," Tenth Annual Biomedical Engineering Research Conference in Houston, March 19-20, 1992.

Rice University Frank Tittel, Ph.D.

\section{A.1 Reflectance spectroscopy}

Our project is to develop spectroscopic applications of diode lasers in the Neonatal Intensive Care Unit (NICU) in collaboration with UTMDA-LL and UTMSH-Ped. The basic areas of application have been described above in the UTMDA report section. We are concentrating on the development of a novel approach toward reflectance spectroscopy: phase and demedulation reflectance spectroscopy.

The basic concept is to modulate a light source at high frequencies in the range of 100 $\mathrm{MHz}$ to $1 \mathrm{GHz}$, which launches photon density waves into the tissue. These photon density waves have wavelengths on the order of $\mathrm{cm}$ and wave velocities on the order of $1 / 10$ the speed of light. The detection sysiem monitors the phase (P) and demodulation (M) of the density waves. 
The analysis of the two data, $\mathrm{P}$ and $\mathrm{M}$, by radiative transport theory yields two unknowns, absorption $\left(\mu_{\mathrm{a}}\right.$ in $\left.\mathrm{cm}^{-1}\right)$ and reduced scattering $\left(\mu_{\mathrm{s}}(1-\mathrm{g})\right.$ in $\left.\mathrm{cm}^{-1}\right)$, for a given modulation frequency and an given spacing between source and detector optical fibers. Therefore, absorption spectroscopy can be achieved which accounts for light scattering in turbid tissues such as skin or brain, yielding values for $\mu a$ which are directly proportional to the concentration of the chromophore of interest (eg., bilirubin, oxyhemoglobin, deoxyhemoglobin).

Our DOE funds are supporting one graduate student (Andrew Hilscher) who is constructing a phase and demodulation reflectance spectroscopy system. The system will incorporate three diode lasers in the $760-830 \mathrm{~nm}$ range, to allow specification of three unknowns: $\mu_{\mathrm{a}}$ background tissue, $\mu_{\mathrm{a}}$ oxyhemoglobin, and $\mu_{\mathrm{a}}$ deoxyhemoglobin.

Our first application will be the monitoring of brain oxygenation in newborn infants. Detection of compromised brain oxygenation immediately after birth can allow surgical intervention and prevent permanent brain damage. Currently, such compromised brain oxygenation is difficult to detect and is not usually evident until several days after birth when brain damage has already occured.

\section{Naval Research Laboratory Leon Esterowitz, Ph.D.}

The research efforts of our group at the Naval Research Laboratory (NRL) have concentrated on the design and development of a compact and portable $2-\mu \mathrm{m}$ solid-state laser system. We have designed a flexible laser system which can be pumped either by a Ti:sapphire laser or by an $\mathrm{AlGaAs}$ diode laser array. The Ti:sapphire-pumped 3- $\mu \mathrm{m}$ laser is a the more short term development effort, allowing full utilization of the Ti:sapphire currently available in the laboratory of A.J. Welch at the University of Texas at Austin. We are developing in parallel a diode-pumped $2-\mu \mathrm{m}$ laser which utilizes the efficiency and convenience of fiber coupling of the laser diode pump radiation.

The $2-\mu \mathrm{m}$ solid-state laser development is based on the research initiated in our laboratory on diode pumping of laser crystals singly-doped with the trivalent thulium ion. This system utilizes the strong absorption by the thulium ion of pump radiation in the $800-\mathrm{nm}$ spectral region, and avoids the upconversion losses associated with co-dopant ions such as erbium and hormium. The thulium system utilizes the two-for-one self-quenching process which excites two ions into the upper laser state for every ion excited by the $800-\mathrm{nm}$ pump laser. This scheme has resulted in laser slope efficiencies as high as $60 \%$, and has produced the highest $2-\mu \mathrm{m}$ output power with diode laser pumping.

For both the Ti:sapphire-pumped and diode-laser-pumped $2-\mu \mathrm{m}$ laser systems we have implemented a water-cooled laser head for removing heat from the laser crystal. This minimizes the temperature rise in the thulium crystal, which is a crucial consideration for a quasi three level laser system such as thulium. In a three-level laser the threshold for laser oscillation increases dramatically as the temperature rises, owing to the increased thermal population in the lower laser state. Therefore it is important to extract heat efficiently from the laser crystal. The water-cooled laser head will allow us to scale up the $2-\mu \mathrm{m}$ laser power for both the Ti:sapphire pumped and diode-laser pumped systems. 
The Ti:sapphire-pumped thulium laser system is currently being installed and set up by NRL personnel in the laboratory of A.J. Welch at UTA. In the coming year this system will be scaled to higher output power, and modified to allow tunable laser output over the range 1.9 to $2.1 \mu \mathrm{m}$.

The ultimate goal of our development effort is a $2-\mu \mathrm{m}$ thulium laser system pumped by fiber coupled laser diode arrays. This system will be compact, rugged, portable, and efficient. We have designed and implemented a laser system utilizing the 5 -watt $\mathrm{cw}$ diode laser arrays currently available. This system makes use of short focal length input optics which produce a tightly focused pump beam from the fiber output. A tightly focused pump beam is necessary in order to minimize the threshold for the relatively low-grain thulium laser material. The small pump spot size is also required to allow the greatest degree of tunability over the 1.9 to $2.1 \mu \mathrm{m}$ spectral range. This laser system also utilizes the water-cooled laser crystal which will allow scale-up to higher $2-\mu \mathrm{m}$ output powers. 

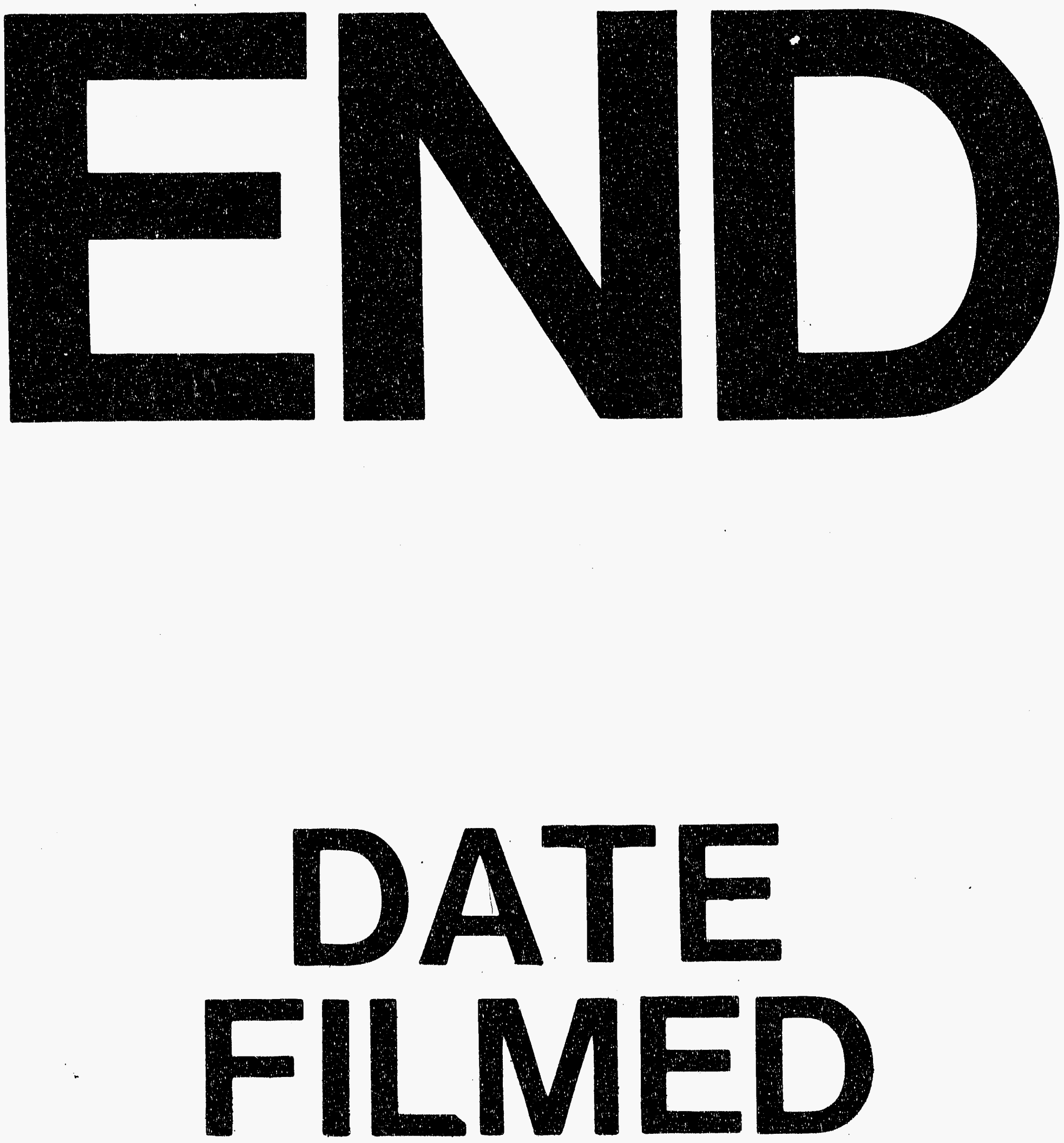

1

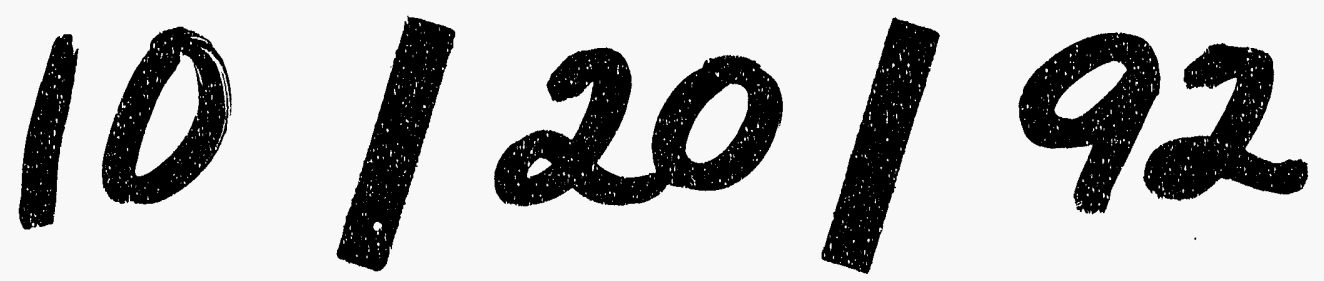


